\title{
Microwave Device Modeling Using Space-Mapping and Radial Basis Functions
}

\author{
Slawomir Koziel, Member, IEEE, and John W. Bandler, Life Fellow, IEEE \\ Department of Electrical and Computer Eng., McMaster University, Hamilton, ON, Canada L8S 4K1
}

\begin{abstract}
A novel surrogate modeling methodology is presented that utilizes space mapping combined with radial basis function interpolation. The method has advantages both over the standard space mapping modeling methodology and the recently published space mapping modeling with variable weight coefficients. In particular, it provides accuracy comparable or better than the latter method and computational efficiency as good as the standard space mapping modeling procedure. Examples illustrating the performance of the new modeling method as well as a comparison with previously published approaches are given.
\end{abstract}

Index Terms - Computer-aided design (CAD), EM modeling, space mapping, surrogate modeling, radial basis functions.

\section{INTRODUCTION}

Full-wave EM simulations of microwave structures are CPU intensive. Statistical analysis and yield optimization, crucial for manufacturability-driven designs in a time-tomarket development environment, demand accurate and fast models. The space mapping (SM) concept [1] addresses this issue.

SM assumes "fine" and "coarse" models. The "fine" model may be a high fidelity CPU-intensive EM simulator, undesirable for direct statistical analysis and design. The "coarse" model can be a simplified representation such as an equivalent circuit with empirical formulas. SM modeling [2]-[6] exploits the speed of the coarse model and the accuracy of the fine model to develop fast, accurate enhanced models (surrogates) valid over a wide range of parameter values.

The standard SM modeling approach is based on setting up the surrogate model using a small amount of fine-model data (usually a so-called star distribution: $2 n+1$ points, where $n$ is the number of design variables) and performing extraction of model parameters over the whole set of this data [4], [5]. This simple methodology gives reasonable accuracy especially for low-dimensional problems. In order to further improve the modeling performance one needs to involve more fine model

This work was supported in part by the Natural Sciences and Engineering Research Council of Canada under Grants OGP0007239 and STPGP336760, and by Bandler Corporation.

S. Koziel is with the Simulation Optimization Systems Research Laboratory, Department of Electrical and Computer Engineering, McMaster University, Hamilton, ON, Canada L8S 4K1.

J.W. Bandler is with the Simulation Optimization Systems Research Laboratory, Department of Electrical and Computer Engineering, McMaster University, Hamilton, ON, Canada L8S 4K1 and also with Bandler Corporation, Dundas, ON, Canada L9H 5E7. information. Unfortunately, SM is not suitable for handling a large amount of fine model data by itself, i.e., increasing the number of base points does not help if the number of degrees of freedom of the model remains unchanged.

A recently published space mapping modeling technique with variable weight coefficients [6] aimed at overcoming these limitations. It provides better accuracy than the standard method, however, at the expense of significant increase of the evaluation time, which is due to a separate parameter extraction required for each evaluation of the surrogate model. This limits potential applications of the method.

In this paper, we present a new approach that combines the standard space mapping modeling methodology with radial basis function interpolation. This combination gives modeling accuracy comparable to or better than the method [6] without compromising computational cost.

\section{SURRogate Modeling WITH SPACE MAPPING AND RADIAL BASIS FUNCTION INTERPOLATION}

Let $R_{f}: X_{f} \rightarrow R^{m}$ and $R_{c}: X_{c} \rightarrow R^{m}$ denote the fine and coarse model response vectors, where $X_{f} \subseteq R^{n}$ and $X_{c} \subseteq R^{n}$ are design variable domains of the fine and coarse models, respectively. For example, $R_{f}(x)$ and $R_{c}(x)$ may represent the magnitude of a transfer function at $m$ chosen frequencies.

We denote by $X_{R} \subseteq X_{f}$ the region of interest in which we want enhanced matching between the surrogate and the fine model. We assume that $X_{R}$ is an $n$-dimensional interval in $R^{n}$ with center at reference point $x^{0}=\left[\begin{array}{lll}x_{0.1} & \ldots & x_{0 . n}\end{array}\right]^{T} \in R^{n}$ :

$$
X_{R}=\left[\boldsymbol{x}^{0}-\boldsymbol{\delta}, \boldsymbol{x}^{0}+\boldsymbol{\delta}\right]=\left[x_{0.1}-\delta_{1}, x_{0.1}+\delta_{1}\right] \times \ldots \times\left[x_{0, n}-\delta_{n}, x_{0 . n}+\delta_{n}\right]
$$

where $\delta=\left[\begin{array}{lll}\delta_{1} & \ldots & \delta_{n}\end{array}\right]^{T}$ determines the size of $X_{R}$. We use $X_{R}\left(x^{0}, \delta\right)$ to denote the region of interest defined by $x^{0}$ and $\delta$. Let $X_{B}=\left\{\boldsymbol{x}^{1}, \boldsymbol{x}^{2}, \ldots, \boldsymbol{x}^{N}\right\} \subset X_{R}\left(\boldsymbol{x}^{0}, \boldsymbol{\delta}\right)$ be the base set, where $N$ is the number of base points, such that the fine model response is known at all points $\boldsymbol{x}^{j}, j=1,2, \ldots, N$. We do not assume any particular location of the base points.

We define a generic surrogate model $\bar{R}_{s}: X_{R} \times M_{m \times m}$

$$
\begin{aligned}
\times M_{n \times n} \times M_{n \times 1} \rightarrow R^{m} \text { as } & \\
& \bar{R}_{s}(\boldsymbol{x}, A, B, \boldsymbol{c})=\boldsymbol{A} \cdot \boldsymbol{R}_{c}(\boldsymbol{B} \cdot \boldsymbol{x}+\boldsymbol{c})
\end{aligned}
$$

with matrices $A=\operatorname{diag}\left\{a_{1}, \ldots, a_{m}\right\}, B \in M_{n \times n}$, and $c \in M_{n \times 1}$ ( $M_{k \times l}$ denotes the set of $k \times l$ real matrices) found using the parameter extraction process

$$
(A, B, c)=\arg \min _{(\alpha, \beta, \gamma)} \sum_{k=1}^{N}\left\|R_{f}\left(x^{k}\right)-\bar{R}_{s}\left(x^{k}, \alpha, \beta, \gamma\right)\right\|
$$


Apart from model (2), (3), an optional frequency scaling can be implemented that works in such a way that the coarse model is evaluated at a different frequency than the fine model using the transformation: $\omega \rightarrow f_{0}+f_{1} \omega$, where $F=\left[f_{0} f_{1}\right] \in R^{2}$ is obtained together with matrices $A, B$, and $c$ using a parameter extraction process similar to (3). More general space mapping surrogate models can be found in [4]-[7].

On the top of the standard SM surrogate, we use radial basis function (RBF) interpolation [8], [9] of the difference between the fine model $R_{f}$ and standard surrogate $\bar{R}_{s}$, denoted

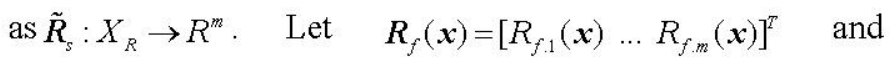
$\bar{R}_{s}(\boldsymbol{x})=\left[\begin{array}{llll}\bar{R}_{s, 1}(\boldsymbol{x}) & \ldots & \bar{R}_{s, m}(\boldsymbol{x})\end{array}\right]^{T} \cdot \tilde{R}_{s}$ is defined as

$$
\tilde{R}_{s}(\boldsymbol{x})=\left[\begin{array}{c}
\sum_{j=1}^{N} \lambda_{1, j} \phi\left(\left\|\boldsymbol{x}-\boldsymbol{x}^{j}\right\| / \gamma\right) \\
\ldots \\
\sum_{j=1}^{N} \lambda_{m, j} \phi\left(\left\|\boldsymbol{x}-\boldsymbol{x}^{j}\right\| / \gamma\right)
\end{array}\right]
$$

where $\|$.$\| denotes the Euclidean norm. The parameters \lambda_{k, j}$ are calculated so that they satisfy

$$
\Phi \lambda_{k}=F_{k} \quad k=1,2, \ldots, m
$$

where $\boldsymbol{\lambda}_{k}=\left[\begin{array}{llll}\lambda_{k .1} & \lambda_{k .2} & \ldots & \lambda_{k . N}\end{array}\right]^{T}$,

$$
F_{k}=\left[\begin{array}{c}
R_{f, k}\left(\boldsymbol{x}^{1}\right)-\bar{R}_{s, k}\left(\boldsymbol{x}^{1}\right) \\
\vdots \\
R_{f, k}\left(\boldsymbol{x}^{N}\right)-\bar{R}_{s, k}\left(\boldsymbol{x}^{N}\right)
\end{array}\right]
$$

and $\Phi$ is $N \times N$ matrix with elements

$$
\Phi_{i j}=\phi\left(\left\|\boldsymbol{x}^{i}-\boldsymbol{x}^{j}\right\| / \gamma\right)
$$

$\gamma=\chi(\delta, N)$ is a characteristic distance of the base set defined as

$$
\gamma(\boldsymbol{\delta}, N)=\frac{2}{n N^{1 / n}} \sum_{i=1}^{n} \delta_{i}
$$

Parameter $\gamma$ is, in fact, an average distance between base points and it is used in (4) as a normalization factor.

In this paper we use a Gaussian basis function defined as

$$
\phi(r)=e^{-c r^{2}} \quad r \geq 0 \quad c>0
$$

Other choices of basis functions can be found in the literature [9].

The combined surrogate model $R_{s}: X_{R} \rightarrow R^{m}$ is defined as

$$
\boldsymbol{R}_{s}(\boldsymbol{x})=\overline{\boldsymbol{R}}_{s}(\boldsymbol{x})+\tilde{\boldsymbol{R}}_{s}(\boldsymbol{x})
$$

Once coefficients $\lambda$ are found, evaluation of (4) is very fast, which means that evaluation cost of model (10) is not significantly larger than evaluation cost of the standard space mapping surrogate model (2). This is in contrast with the modeling technique [6] requiring a separate parameter extraction for each evaluation of the surrogate model, which involves a number of coarse model evaluations (typically hundreds or even thousands).

\section{EXAMPLES}

In this section, we compare the modeling accuracy for the standard SM modeling methodology [4], SM modeling with variable weight coefficients [6], and the new modeling approach (2)-(10) described in Section II.

\section{A. Test Problem Description}

Problem 1: Two-section capacitively-loaded impedance transformer [10]. The coarse model and the fine model are, respectively, an ideal two-section transmission line (TL) and a capacitively-loaded TL as shown in Fig. 1. Both models are implemented in Matlab. The design parameters are $\boldsymbol{x}=\left[L_{1} L_{2}\right]^{T}$. The frequency range is $0.5 \mathrm{GHz} \leq \omega \leq 1.5 \mathrm{GHz}$. The reference point is $x^{0}=\left[74.25^{\circ} 79.24^{\circ}\right]^{T}$. The region of interest is defined by a $10 \%$ deviation from $x^{0}$. We consider $\left|S_{11}\right|$ as the model response.

Problem 2: Seven-section capacitively-loaded impedance transformer [10]. The coarse and fine models are shown in Fig. 2 (both implemented in Matlab). The frequency range is $1.0 \mathrm{GHz} \leq \omega \leq 7.7 \mathrm{GHz}$. The design parameters are $\boldsymbol{x}=\left[L_{1} L_{2} L_{3} L_{4} L_{5} L_{6} L_{7}\right]^{T}$. The reference point is $x^{0}=\left[\begin{array}{lllllllll}1 & 1 & 1 & 1 & 1 & 1 & 1\end{array}\right]^{T}$. We consider the region of interest defined by a $5 \%$ deviation from $x^{0}$.

Problem 3: Microstrip right-angle bend [3]. The fine model, Fig. 3(a), is analyzed by Sonnet's $\mathbf{e m}^{\mathrm{TM}}$. The coarse model, Fig. 3(b), is an equivalent circuit with parameters calculated from Kirschning et al. [12]. The design parameters are $\boldsymbol{x}=\left[\begin{array}{lll}W & H & \varepsilon_{r}\end{array}\right]^{T}$. The frequency range is 1 to $31 \mathrm{GHz}$. The reference point is $x^{0}=\left[\begin{array}{lll}25 & 12 & 9\end{array}\right]^{T}$, and the region size is $\delta=\left[\begin{array}{lll}6 & 4 & 1\end{array}\right]^{T}$.

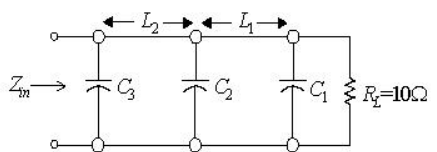

(a)

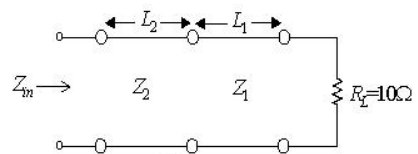

(b)
Fig.1. Two-section capacitively-loaded impedance transformer [10]: "fine" model (a) and "coarse" model (b).

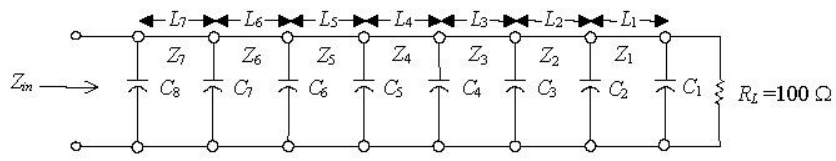

(a)

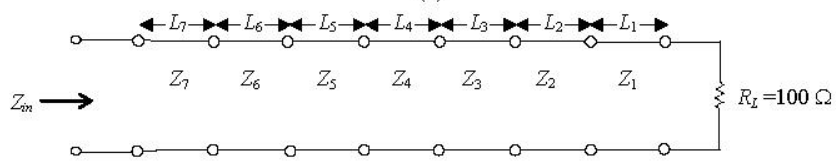

(b)

Fig. 2. Seven-section capacitively-loaded impedance transformer [10]: "fine" model (a) and "coarse" model (b).

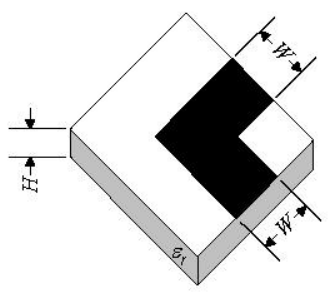

(a)

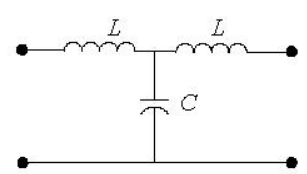

(b)
Fig.3. The microstrip right-angle bend [3]: the fine model (a) and the coarse model (b). 


\section{B. Experimental Setup}

For each of the test problems, we performed a number of experiments using the standard SM surrogate model, SM model [6], pure RBF interpolation (i.e., RBF interpolating directly to fine model data) as well as the new SM model (2)-(10). Table I shows details of the base sets used in our experiments. The base sets have growing numbers of points (and decreasing characteristic distances $\gamma$ ) in order to examine the dependence of the modeling error on the amount of fine model data used to create the model. The standard SM model uses the star-distribution base set [4] and surrogate model (2) enhanced by frequency space mapping.

Accuracy was tested using 30 test points randomly distributed in the region of interest. The error measure used was the $h_{2}$ norm of the difference between the fine model response and the corresponding surrogate model response.

TABLE I

Base Set Data For TeSt PROBLEMS 1-3

\begin{tabular}{|c|c|c|c|c|}
\hline $\begin{array}{c}\text { Test } \\
\text { Problem }\end{array}$ & $\begin{array}{c}\text { Base } \\
\text { Set }\end{array}$ & $\begin{array}{c}\text { Base Set } \\
\text { Description }\end{array}$ & $\begin{array}{l}\text { Number of } \\
\text { Base Points }\end{array}$ & $\gamma$ \\
\hline \multirow{4}{*}{1} & $X_{B 1}$ & Uniform mesh of density 3 & 9 & 5.12 \\
\hline & $X_{B 2}$ & Uniform mesh of density 5 & 25 & 3.07 \\
\hline & $X_{B 3}$ & Uniform mesh of density 7 & 49 & 2.19 \\
\hline & $X_{B 4}$ & Uniform mesh of density 10 & 100 & 1.53 \\
\hline \multirow{3}{*}{2} & $X_{B 1}$ & Star distribution & 15 & 0.136 \\
\hline & $X_{B 2}$ & Uniform mesh of density $2+x^{0}$ & 129 & 0.100 \\
\hline & $X_{B 3}$ & Uniform mesh of density 3 & 2187 & 0.067 \\
\hline \multirow{4}{*}{3} & $X_{B 1}$ & Uniform mesh of density 2 & 8 & 3.66 \\
\hline & $X_{B 2}$ & Uniform mesh of density 3 & 27 & 2.44 \\
\hline & $X_{B 3}$ & Uniform mesh of density 4 & 64 & 1.83 \\
\hline & $X_{B 4}$ & Uniform mesh of density 5 & 125 & 1.47 \\
\hline
\end{tabular}

\section{Experimental Results and Discussion}

Tables II, III, and IV show numerical results (error statistics) for the standard SM model, SM model [6], pure radial basis function interpolation, and the new model (2)-(10) with all the base sets considered. Figs. 4-6 show dependence of average modeling error on the characteristic distance $\gamma$. Table $\mathrm{V}$ presents the qualitative comparison of the surrogate model evaluation cost for all the considered methods.

The results show that the new model-the combination of space mapping and RBF interpolation-outperforms both the standard SM model and the SM model with variable weight coefficients [6], as well as the pure RBF interpolation. Moreover, the computational cost of the new method is significantly lower than the cost of the SM model [6] and almost the same as for the standard SM model. The pure RBF interpolation is the fastest method because it only requires evaluation of formula (4), however, it cannot compete with the new model (2)-(10) in terms of modeling accuracy.
TABLE II

MODELING RESUlts For TEST PROBLEM 1

VERIFICATION FOR 30 RANDOM TEST POINTS

\begin{tabular}{ccccc}
\hline \hline Model & Base & Average & Maximum & Standard \\
Description & Set & Error & Error & Deviation \\
\hline Standard SM Model & star dist. & 0.0404 & 0.0703 & 0.0130 \\
SM Model [6] & $X_{B 1}$ & 0.0218 & 0.0446 & 0.0080 \\
SM Model [6] & $X_{B 2}$ & 0.0133 & 0.0330 & 0.0064 \\
SM Model [6] & $X_{B 3}$ & 0.0087 & 0.0244 & 0.0048 \\
SM Model [6] & $X_{B 4}$ & 0.0058 & 0.0220 & 0.0043 \\
Pure RBF Interpolation & $X_{B 1}$ & 0.0310 & 0.0642 & 0.0165 \\
Pure RBF Interpolation & $X_{B 2}$ & 0.0073 & 0.0181 & 0.0045 \\
Pure RBF Interpolation & $X_{B 3}$ & 0.0042 & 0.0189 & 0.0044 \\
Pure RBF Interpolation & $X_{B 4}$ & 0.0033 & 0.0124 & 0.0028 \\
New SM Model (2)-(10) & $X_{B 1}$ & 0.0134 & 0.0259 & 0.0059 \\
New SM Model (2)-(10) & $X_{B 2}$ & 0.0057 & 0.0154 & 0.0036 \\
New SM Model (2)-(10) & $X_{B 3}$ & 0.0031 & 0.0067 & 0.0016 \\
New SM Model (2)-(10) & $X_{B 4}$ & 0.0016 & 0.0041 & 0.0011 \\
\hline \hline
\end{tabular}

TABLE III

Modeling Results For Test PRoBlem 2 VERIFICATION FOR 30 RANDOM TEST POINTS

\begin{tabular}{ccccc}
\hline \hline Model & Base & Average & Maximum & Standard \\
Description & Set & Error & Error & Deviation \\
\hline Standard SM Model & star dist. & 0.0136 & 0.0232 & 0.0039 \\
SM Model [6] & $X_{B 1}$ & 0.0151 & 0.0299 & 0.0061 \\
SM Model [6] & $X_{B 2}$ & 0.0105 & 0.0150 & 0.0020 \\
SM Model [6] & $X_{B 3}$ & 0.0053 & 0.0082 & 0.0013 \\
Pure RBF Interpolation & $X_{B 1}$ & 0.0821 & 0.2057 & 0.0509 \\
Pure RBF Interpolation & $X_{B 2}$ & 0.0679 & 0.1441 & 0.0244 \\
Pure RBF Interpolation & $X_{B 3}$ & 0.0365 & 0.0788 & 0.0144 \\
New SM Model (2)-(10) & $X_{B 1}$ & 0.0098 & 0.0195 & 0.0039 \\
New SM Model (2)-(10) & $X_{B 2}$ & 0.0088 & 0.0129 & 0.0019 \\
New SM Model (2)-(10) & $X_{B 3}$ & 0.0036 & 0.0057 & 0.0009 \\
\hline \hline
\end{tabular}

TABLE IV

Modeling Results For Test PRoBlem 3 VERIFICATION FOR 30 RANDOM TEST POINTS

\begin{tabular}{|c|c|c|c|c|}
\hline $\begin{array}{c}\text { Model } \\
\text { Description }\end{array}$ & $\begin{array}{l}\text { Base } \\
\text { Set }\end{array}$ & $\begin{array}{l}\text { Average } \\
\text { Error }\end{array}$ & $\begin{array}{l}\text { Maximum } \\
\text { Error }\end{array}$ & $\begin{array}{c}\text { Standard } \\
\text { Deviation }\end{array}$ \\
\hline Standard SM Model & star dist. & 0.0116 & 0.0275 & 0.0062 \\
\hline SM Model [6] & $X_{B 1}$ & 0.0492 & 0.1728 & 0.0389 \\
\hline SM Model [6] & $X_{B 2}$ & 0.0089 & 0.0541 & 0.0096 \\
\hline SM Model [6] & $X_{B 3}$ & 0.0036 & 0.0093 & 0.0020 \\
\hline SM Model [6] & $X_{B 4}$ & 0.0023 & 0.0066 & 0.0014 \\
\hline Pure RBF Interpolation & $X_{B 1}$ & 0.0824 & 0.1050 & 0.0198 \\
\hline Pure RBF Interpolation & $X_{B 2}$ & 0.0306 & 0.0726 & 0.0158 \\
\hline Pure RBF Interpolation & $X_{B 3}$ & & 0.0891 & 0.0219 \\
\hline Pure RBF Interpolation & $X_{B 4}$ & 0.0273 & 0.0337 & 0.0136 \\
\hline New SM Model (2)-(10) & $X_{B 1}$ & 0.0062 & 0.0115 & 0.0028 \\
\hline New SM Model (2)-(10) & $X_{B 2}$ & 0.0013 & 0.0036 & 0.0008 \\
\hline New SM Model (2)-(10) & $X_{B 3}$ & 0.0011 & 0.0027 & 0.0009 \\
\hline New SM Model (2)-(10) & $X_{B 4}$ & 0.0009 & 0.0030 & 0.0006 \\
\hline
\end{tabular}




\section{TABLE V}

QUALITATIVE COMPARISON OF COMPUTATIONAL COST OF EVALUATING SURROGATE MODEL

\begin{tabular}{ccc}
\hline \hline $\begin{array}{c}\text { Modeling } \\
\text { Method }\end{array}$ & $\begin{array}{c}\text { Main Sources of } \\
\text { Computational Cost }\end{array}$ & $\begin{array}{c}\text { Relative Evaluation } \\
\text { Cost }\end{array}$ \\
\hline Standard SM Model & Coarse model evaluation & $\begin{array}{c}\text { Similar to coarse } \\
\text { model } \\
\text { Such higher than } \\
\text { coarse model }\end{array}$ \\
SM Model [6] & Parameter extraction & $\begin{array}{c}\text { Lower than coarse } \\
\text { model } \\
\text { RBF Interpolation }\end{array}$ Evaluation of formula (4) \\
SM Model (2)-(10) & Coarse model evaluation & $\begin{array}{c}\text { Similar to coarse } \\
\text { model }\end{array}$ \\
\hline \hline
\end{tabular}

\section{CONCLUSION}

A new SM-based modeling methodology is presented. It combines space mapping with radial basis function interpolation. This combination allows us to reduce the modeling error to a level not attainable by any other space mapping technique without compromising computational cost.

\section{REFERENCES}

[1] J.W. Bandler, Q.S. Cheng, S.A. Dakroury, A.S. Mohamed, M.H. Bakr, K. Madsen, and J. Sondergaard, "Space mapping: the state of the art," IEEE Trans. Microwave Theory Tech, vol. 52, no. 1, pp. 337-361, Jan. 2004.

[2] M.H. Bakr, J.W. Bandler, and N. Georgieva, "Modeling of microwave circuits exploiting space derivative mapping," IEEE MTT-S Int. Microwave Symp. Dig., Anaheim, CA, June 1999, pp. 715-718.

[3] J.W. Bandler, N. Georgieva, M.A. Ismail, J.E. Rayas-Sánchez, and Q. J. Zhang, "A generalized space mapping tableau approach to device modeling," IEEE Trans. Microwave Theory Tech., vol. 49, no. 1, Jan. 2001, pp. 67-79.

[4] S. Koziel, J.W. Bandler, A.S. Mohamed, and K. Madsen, "Enhanced surrogate models for statistical design exploiting space mapping technology," IEEE MTT-S Int. Microwave Symp. Dig., Long Beach, CA, June 2005, pp. 1609-1612.

[5] J.W. Bandler, Q.S. Cheng, and S. Koziel, "Implementable space mapping approach to enhancement of microwave device models," IEEE MTT-S Int. Microwave Symp. Dig., Long Beach, CA, June 2005, pp. 11391142.

[6] S. Koziel and J.W. Bandler, "Space-mapping-based modeling utilizing parameter extraction with variable weight coefficients and a data base," IEEE MTT-S Int. Microwave Symp. Dig., San Francisco, 2006.

[7] S. Koziel, J.W. Bandler, and K. Madsen, "A space mapping framework for engineering optimization: theory and implementation," IEEE Trans. Microwave Theory Tech., vol. 54, no. 10, pp. 3721-3730, Oct. 2006.

[8] M.J.D. Powell, "Radial basis functions for multivariate interpolation: a review," In Algorithms for Approximation, J.C. Mason and M.G. Cox, Eds. Clarendon Press, Oxford, 1987.

[9] M.D. Buhmann and M. J. Ablowitz, Radial Basis Functions: Theory and Implementations, Cambridge University, 2003.

[10] M.H. Bakr, J.W. Bandler, K. Madsen, and J. Søndergaard, "An introduction to the space mapping technique," Optimization Eng., vol. 2, no. 4, pp. 369-384, Dec. 2001.

[11] M. Kirschning, R. H. Jansen, and N. H. L. Koster, "Measurement and computer-aided modeling of microstrip discontinuities by an improved resonator method," IEEE MTT-S Int. Microwave Symp. Dig., Boston, MA, May 1983, pp. 495-497.

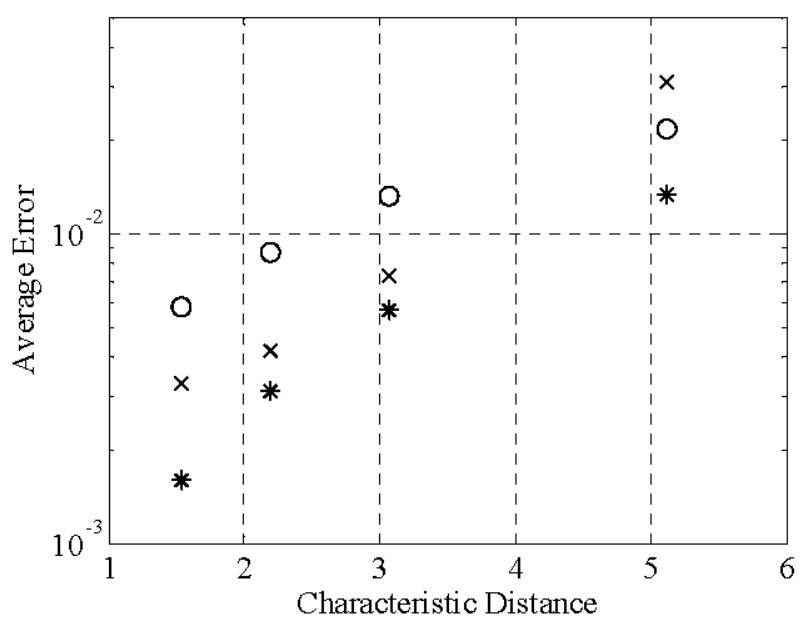

Fig.4. Average modeling error versus characteristic distance $\gamma$ of the base set for test problem 1 (two-section impedance transformer). Data for SM model [6] (o), pure RBF interpolation $(\times)$, and new SM model (2)-(10) (*).

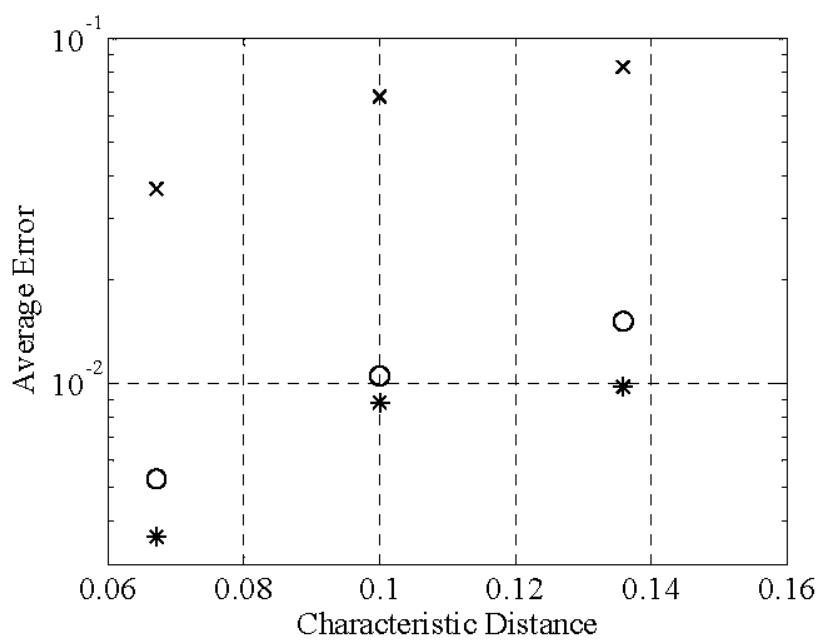

Fig.5. Average modeling error versus characteristic distance $\gamma$ of the base set for test problem 2 (seven-section impedance transformer). Data for SM model [6] (o), pure RBF interpolation ( $\times$ ), and new SM model (2)-(10) (*).

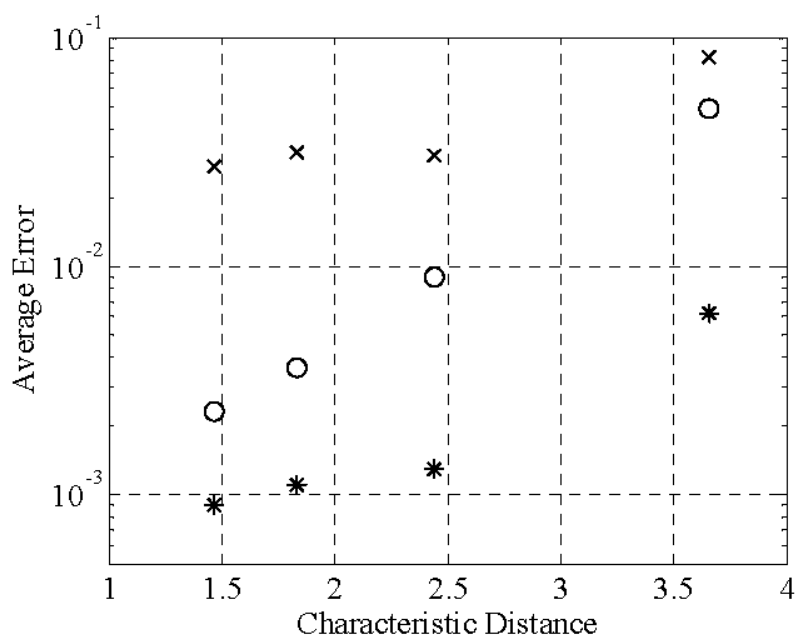

Fig.6. Average modeling error versus characteristic distance $\gamma$ of the base set for test problem 1 (microstrip right-angle bend). Data for SM model [6] (o), pure RBF interpolation $(\times)$, and new SM model $(2)-(10)(*)$. 